\title{
Persistent abnormal left ventricular systolic torsion in dilated cardiomyopathy after partial left ventriculectomy
}

\author{
Randolph M. Setser, DSc ${ }^{a}$ \\ Jane M. Kasper, $\mathrm{RN}^{\mathrm{a}}$ \\ Michael L. Lieber, MS ${ }^{b}$ \\ Randall C. Starling, MD ${ }^{c}$ \\ Patrick M. McCarthy, MD \\ Richard D. White, MD ${ }^{a, c, d}$
}

From the Departments of Radiology ${ }^{\mathrm{a}}$ (Section of Cardiovascular Imaging), Biostatistics and Epidemiology, ${ }^{\mathrm{b}}$ Cardiovascular Medicine, ${ }^{\mathrm{c}}$ and Thoracic and Cardiovascular Surgery, ${ }^{\mathrm{d}}$ The Cleveland Clinic Foundation, Cleveland, Ohio.

Received for publication June 21, 2002; revisions requested Aug 1, 2002; revisions received Oct 15, 2002; accepted for publication Oct 17, 2002.

Address for reprints: Randolph M. Setser, DSc, Division of Radiology, Desk Hb6, The Cleveland Clinic Foundation, 9500 Euclid Ave, Cleveland, OH 44195 (E-mail: setserr@ccf.org).

J Thorac Cardiovasc Surg 2003;126:48-55

Copyright $(\odot) 2003$ by The American Association for Thoracic Surgery

$0022-5223 / 2003 \$ 30.00+0$

doi:10.1016/S0022-5223(03)00050-3
Objective: Rotation of the left ventricular apex relative to the base, or left ventricular torsion, is related to myocardial contractility and structure and is a sensitive indicator of cardiac dysfunction. We have quantified left ventricular systolic rotation and torsion in patients with dilated cardiomyopathy before and after partial left ventriculectomy in an attempt to characterize the effects of this surgical procedure on ventricular ejection mechanics.

Methods: Magnetic resonance imaging with tissue tagging was performed before partial left ventriculectomy in 24 patients, 9 of whom underwent repeat imaging 3 months after surgery. Left ventricular rotation was quantified in each patient at three short-axis levels: apex, midventricle, and base. Torsion was defined as the difference between basal and apical rotation at any time. Results were subdivided for regional analysis at each level and related to cardiac function (ejection fraction, cardiac index, and velocity of circumferential fiber shortening).

Results: Before surgery, left ventricular rotation was regionally heterogeneous and abnormal in magnitude and pattern, and increased end-systolic torsion was associated with better cardiac function. After surgery, clinical indices of cardiac function showed improvement; however, rotation magnitude was unchanged at the apex and reduced at the base and midventricle, particularly in the anterior wall and septum.

Conclusions: The pattern and magnitude of ventricular rotation were impaired by dilated cardiomyopathy. Left ventricular rotation and torsion were further diminished after partial left ventriculectomy, indicating that improvement in clinical indices of cardiac function was not reflective of an improvement in this measure of myocardial mechanics.

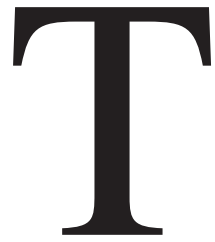

he mammalian left ventricle (LV) consists of obliquely oriented muscle fibers that vary from a right-handed helix at the subendocardium to a left-handed helix at the subepicardium, with most myofibers in an approximately circumferential orientation. ${ }^{1}$ The functional results of this 3-dimensional structure are cyclic twisting and untwisting of the LV apex relative to the base (torsion) that accompany contraction and relaxation, respectively, and are thought to equalize fiber stress and sarcomere length across the LV wall. ${ }^{2,3}$

Studies of LV torsion have shown that in healthy hearts all short-axis levels from base to apex rotate counterclockwise, when viewed from the apex, during isovolumic contraction. ${ }^{4}$ This is followed by a divergence of basal and apical rotation, with the apex continuing counterclockwise while the base reverses direction and rotates clockwise during ejection. Rotation in intermediate levels varies smoothly between that exhibited at the base and apex. ${ }^{4}$ Furthermore, alterations in the pattern or magnitude of LV rotation and torsion have been associated with cardiovascular disease. $^{5-9}$ 
It has been shown that myofiber orientation is preserved in patients with dilated cardiomyopathy (DCM) ${ }^{10}$ Cardiac function is significantly impaired, however, and the extracellular matrix undergoes significant changes as the disease progresses. $^{11}$

Partial left ventriculectomy (PLV), pioneered by Batista and colleagues ${ }^{12}$ as a treatment for end-stage heart failure, involves excision of a significant portion of the LV free wall in an attempt to reduce LV wall stress and bring geometry back toward normal. Clinical results with this procedure have been variable, with high mortality and few a priori predictors of patient outcome, ${ }^{13,14}$ and thus it has been discontinued at most institutions, including our own. Many studies of clinical indices of ventricular function have been performed in patients after this procedure, ${ }^{13,15}$ and several modeling studies have attempted to predict alterations in LV mechanics after PLV. ${ }^{16,17}$ However, few studies have examined the functional consequences of PLV with respect to ventricular mechanics. ${ }^{7}$ The aims of this study were therefore to characterize the mechanics of ventricular ejection in patients with DCM by quantifying the time course of LV systolic rotation and torsion and to quantify the effects of PLV surgery on LV rotation and torsion in these patients.

\section{Methods \\ Patients}

Twenty-four patients with DCM (15 male, age $55 \pm 13$ years) undergoing magnetic resonance imaging (MRI) for surgical planning before PLV at the Cleveland Clinic Foundation between June 1996 and July 1998 (the procedure was discontinued soon after) were included in this study $(16 \pm 24$ days between baseline MRI and PLV). All patients had previously diagnosed DCM of either idiopathic $(n=18)$, valvular $(n=2)$, or familial $(n=1)$ origin, and all had New York Heart Association functional class III ( $\mathrm{n}=$ $12)$ or class IV $(n=12)$ symptoms of heart failure.

Ten patients returned for follow-up MRI examination approximately 3 months after PLV ( $97 \pm 22$ days between PLV and postoperative MRI). The remaining patients did not undergo follow-up MRI either for clinical reasons, including death or placement of an LV assist device $(\mathrm{n}=8)$, because of prohibitive travel distance $(n=5)$, or because of new absolute contraindications to the technique, such as placement of a permanent pacemaker $(n=1)$.

All data obtained for this study were reviewed and approved by the Cleveland Clinic Foundation institutional review board. All MRI examinations were clinically indicated and were conducted according to an institutional review board-approved protocol with approved waivers of individual consent.

\section{Surgical Procedure}

The surgical technique used in the PLV procedure has been described elsewhere. ${ }^{18}$ Briefly, a wedge-shaped section of the basal to middle LV free wall between the papillary muscles, supplied by the circumflex coronary artery, was excised in all 24 patients. This was accompanied by mitral valve repair in 23 cases, DeVega tricuspid valve annuloplasty ${ }^{19}$ in 11 cases, resection and resuspen- sion of papillary muscles in 11 cases, coronary artery bypass grafting in 2 cases, and mitral valve replacement in 1 case (Table 1).

\section{Imaging}

MRI examinations were performed with a 1.5-T whole-body scanner (Magnetom Vision; Siemens Medical Systems, Erlangen, Germany). After conventional scout imaging, dynamic image loops were acquired along the cardiac short axis in contiguous slices from the mitral valve to $L V$ apex with an electrocardiographically triggered, segmented k-space, cine gradient echocardiographic protocol (FLASH, TE $4.8 \mathrm{~ms}$, sequence TR $100 \mathrm{~ms}$, flip angle $20^{\circ}$, slice thickness 8-10 mm, field of view 300-360 mm with 6/8-8/8 rectangular matrix starting with $256 \times 256$ lines). Temporal resolution was $50 \mathrm{~ms}$ with view sharing. ${ }^{20}$ For patients capable of repetitive 6- to 10-second breath holds, a single signal average was used. Otherwise, three averages were used during free breathing at each level. Dynamic tagged short-axis image loops were acquired at the locations of the cine images with an electrocardiographically triggered, segmented k-space, gradient echo grid tagged imaging protocol (Spatial Modulation of Magnetization, grid tag line spacing $8 \mathrm{~mm}$, with TE $4 \mathrm{~ms}$, sequence TR $90 \mathrm{~ms}$, flip angle $15^{\circ}$, temporal resolution $45 \mathrm{~ms}$, with field of view and matrix parameters matching the cine gradient echo sequence). ${ }^{21}$ Conventional dynamic phase-contrast imaging was used to measure instantaneous volume-flow through the ascending aorta over the cardiac cycle (acquisition parameters TE $5 \mathrm{~ms}$, TR $24 \mathrm{~ms}$, flip angle $30^{\circ}$, slice thickness $6 \mathrm{~mm}$, field of view 300-350 mm, 6/8-8/8 rectangular matrix starting with $128 \times 256$ lines, and three signal averages). ${ }^{22}$

\section{Data Analysis}

Image analysis was performed on an Argus cardiovascular image analysis workstation (v2.0; Siemens Corporate Research, Princeton, NJ).

In cine images at all short-axis levels, the LV myocardium was delineated at end-diastole and end-systole. The mass and blood pool volume of each slice were computed as the area between the endocardial and epicardial contours and within the endocardial contour, respectively, multiplied by the slice thickness. LV mass and LV volumes at end-diastole and end-systole were computed by summing individual slice values.

Three LV short-axis levels were identified for systolic function analysis in each patient. The midventricular level was defined first with image loops in which papillary muscle insertion points were visualized throughout the cardiac cycle. The basal level was defined by the image loops closest to the mitral annulus in which no valve plane or membranous septum was visualized at any point during the cardiac cycle. Apical level image loops were found at approximately $50 \%$ of the distance between midventricle and LV apex in which sufficient myocardial thickness was present for analysis and the right ventricle was visible.

At midventricle, the mean velocity of circumferential fiber shortening $\left(\mathrm{V}_{\mathrm{cf}}\right)$ was computed as $\mathrm{V}_{\mathrm{cf}}=\% \Delta \mathrm{D} / \mathrm{LVET}$, where $\% \Delta D$ is percentage fractional shortening and LVET is ejection time estimated from MRI image loops. ${ }^{23}$

Each data set was subdivided for regional tagging analysis, according to individual patient anatomy (Figure 1). Basal and midventricular levels were each divided into four regions: anterior, inferior, 
TABLE 1. LV morphologic and functional status in patients at baseline, with surgical interventions performed for each patient for whom baseline MRI data were acquired

\begin{tabular}{|c|c|c|c|c|c|c|c|c|c|c|}
\hline Patient & $\begin{array}{l}\text { Age } \\
\text { (y) }\end{array}$ & Sex & NYHA & $\begin{array}{l}\text { LVM } \\
\text { (g) }\end{array}$ & $\begin{array}{l}\text { EDV } \\
(\mathrm{mL})\end{array}$ & $\begin{array}{c}\mathrm{EF} \\
(\%)\end{array}$ & $\begin{array}{l}\text { MRF } \\
(\%)\end{array}$ & $\begin{array}{c}\mathrm{Cl} \\
\left(\mathrm{L} /\left[\mathrm{min} \cdot \mathrm{m}^{2}\right]\right)\end{array}$ & $\begin{array}{c}\mathrm{V}_{\mathrm{cf}} \\
\text { (circ/s) }\end{array}$ & $\begin{array}{c}\text { Surgical } \\
\text { interventions }\end{array}$ \\
\hline 1 & 47 & $\mathrm{M}$ & 4 & 339 & 315 & 12 & - $^{*}$ & 2.0 & 0.06 & 1 \\
\hline 2 & 67 & $\mathrm{~F}$ & 3 & 522 & 621 & 11 & 一 $^{*}$ & 3.4 & 0.31 & 1 \\
\hline 3 & 61 & $\mathrm{M}$ & 3 & 320 & 266 & 21 & 38 & 1.9 & 0.33 & 1,2 \\
\hline 4 & 50 & $\mathrm{M}$ & 3 & 437 & 536 & 19 & 38 & 4.8 & 0.31 & 1,3 \\
\hline 5 & 58 & $\mathrm{M}$ & 3 & 555 & 737 & 8 & 0 & 3.1 & 0.11 & $1,2,3$ \\
\hline 6 & 46 & $\mathrm{~F}$ & 4 & 237 & 176 & 41 & -21 & 2.9 & 0.82 & 1,3 \\
\hline 7 & 57 & $\mathrm{M}$ & 3 & 345 & 337 & 12 & 21 & 2.2 & 0.18 & 1,2 \\
\hline 8 & 34 & $\mathrm{M}$ & 4 & 453 & 621 & 9 & 54 & 3.0 & 0.09 & 1,2 \\
\hline 9 & 66 & $\mathrm{M}$ & 3 & 281 & 292 & 12 & 50 & 2.7 & 0.08 & $1,3,4$ \\
\hline 10 & 62 & $\mathrm{M}$ & 4 & 499 & 541 & 14 & 0 & 2.3 & 0.30 & 1 \\
\hline 11 & 52 & $\mathrm{M}$ & 4 & 614 & 613 & 11 & 50 & 3.3 & 0.68 & 1,2 \\
\hline 12 & 62 & $\mathrm{M}$ & 3 & 420 & 319 & 41 & 56 & 4.7 & 0.20 & 1,3 \\
\hline 13 & 67 & $\mathrm{M}$ & 3 & 377 & 610 & 8 & 47 & 2.7 & 0.80 & $1,2,3$ \\
\hline 14 & 50 & $\mathrm{~F}$ & 3 & 378 & 184 & 33 & 0 & 2.8 & 0.13 & 5 \\
\hline 15 & 62 & $\mathrm{M}$ & 4 & 377 & 327 & 28 & 34 & 3.1 & 0.78 & 1,2 \\
\hline 16 & 71 & $F$ & 4 & 341 & 353 & 11 & - $^{*}$ & 1.7 & 0.62 & $1,2,3$ \\
\hline 17 & 68 & $\mathrm{~F}$ & 3 & 284 & 237 & 20 & — $^{*}$ & 2.5 & 0.58 & 1,2 \\
\hline 18 & 53 & $\mathrm{~F}$ & 4 & 374 & 466 & 12 & 57 & 3.0 & 0.30 & $1,2,3$ \\
\hline 19 & 64 & $\mathrm{M}$ & 3 & 468 & 366 & 25 & 41 & 3.6 & 0.23 & 1,3 \\
\hline 20 & 17 & $\mathrm{M}$ & 4 & 300 & 354 & 47 & 81 & 8.7 & 0.22 & 1,2 \\
\hline 21 & 43 & $M$ & 3 & 541 & 771 & 16 & 74 & 5.5 & 0.21 & 1,3 \\
\hline Mean & 55 & & 3.4 & 403 & 431 & 20 & 36 & 3.3 & 0.35 & \\
\hline SD & 13 & & 0.5 & 102 & 179 & 12 & 28 & 1.6 & 0.25 & \\
\hline$P$ valuet & & & NS & NS & NS & NS & NS & NS & NS & \\
\hline
\end{tabular}

Surgical-interventions were as follows: 1, Mitral valve repair; 2, DeVega tricuspid valve annuloplasty; 3, papillary muscle resection and resuspension; 4, coronary artery bypass grafting; 5 , mitral valve replacement. See text for details. NYHA, New York Heart Association functional class; LVM, LV mass; EDV, LV volume at end-diastole; MRF, mitral regurgitant fraction; $\mathrm{Cl}$, cardiac index; $N S$, not statistically significant.

* Not computed because phase-contrast data were not acquired.

$\dagger P$ values represent comparisons between patients with a post-PLV examination (patients 1-9) and those without a post-PLV examination (patients 10-21).

lateral, and septal. The limits of the septum were defined by the insertion points of the right ventricle, and the lateral wall extended between the papillary muscles, with anterior and inferior wall assignments dependent on those definitions. The apical slice was divided into only two regions, septum and free wall, with the septum defined as myocardium between the right ventricular insertion points.

For grid-tagged short-axis image analysis, epicardial and endocardial contours were traced manually at end-diastole at each level, to define the myocardium and for calculation of slice center of mass. Tag intersection points within the myocardium were then identified automatically by the image analysis software and manually corrected by the operator in all systolic frames (Figure 1). The grid overlay in each frame was reduced to triangular tissue elements, and the centroid of each element relative to the slice center of mass was determined. ${ }^{4}$ The end-diastolic position of each element centroid was used as a reference for rotation analysis. The rotation of any triangular element was defined as the angle between radial lines connecting its centroid with the slice center of mass at end-diastole and any other systolic frame. By convention, counterclockwise rotation was considered positive as viewed from the apex toward the base. ${ }^{4}$

Global rotation was defined as the average rotation of all triangular elements in a slice. Regional rotation was defined as the average rotation of all triangular elements in that region at a particular level. Torsion was defined as the difference in global rotation between the basal and apical levels.
Tissue contained in the lateral wall in basal and midventricular levels at baseline was removed during surgery. This region therefore was excluded from the post-PLV analysis.

The ascending aorta was manually delineated in systolic phasecontrast images to calculate net forward LV volume flow, or effective stroke volume. Mitral regurgitant fraction was calculated as the difference between stroke volume and effective stroke volume divided by stroke volume.

\section{Statistical Analysis}

Comparisons made between baseline and post-PLV values included only results from the 9 patients for whom data were acquired at both time points, and their statistical significance was assessed with the Wilcoxon signed rank test. ${ }^{24}$ The significance of all unpaired comparisons was assessed with the Mann-Whitney rank sum test. ${ }^{24}$

\section{Results}

At baseline, tagged image data were analyzed in 21 of 24 studies. Three data sets were not analyzed either because the MRI examination was prematurely ended at the patient's request $(n=2)$ or because images were not suitable for analysis as a result of inadequate respiratory compensation $(n=1)$. Post-PLV tagged image data were analyzed in 9 of 
TABLE 2. LV morphologic and functional status in patients 3 months after PLV

\begin{tabular}{cccccccc}
\hline Patient & NYHA & $\begin{array}{c}\text { LVM } \\
(\mathbf{g})\end{array}$ & $\begin{array}{c}\text { EDV } \\
(\mathbf{m L})\end{array}$ & $\begin{array}{c}\text { EF } \\
(\%)\end{array}$ & $\begin{array}{c}\text { MRF } \\
(\%)\end{array}$ & $\begin{array}{c}\mathbf{C l} \\
\left(\mathbf{L} /\left[\mathbf{m i n} \cdot \mathbf{m}^{2}\right]\right)\end{array}$ & $\begin{array}{c}\mathbf{V}_{\mathbf{c f}} \\
(\mathbf{c i r c} / \mathbf{s})\end{array}$ \\
\hline 1 & 3 & 220 & 220 & 41 & $-^{*}$ & 3.0 & 0.66 \\
2 & 3 & 465 & 427 & 18 & 22 & 2.9 & 0.31 \\
3 & 2 & 257 & 202 & 31 & 39 & 2.5 & 0.57 \\
4 & 2 & 297 & 272 & 23 & 20 & 3.2 & 0.49 \\
5 & 2 & 381 & 308 & 19 & 0 & 3.0 & 0.28 \\
6 & 3 & 179 & 138 & 41 & 33 & 2.3 & 0.92 \\
7 & 3 & 236 & 170 & 27 & 0 & 2.4 & 0.49 \\
8 & 4 & 313 & 404 & 20 & 0 & 3.6 & 0.35 \\
9 & 3 & 321 & 322 & 17 & 23 & 2.3 & 0.3 \\
Mean & 2.8 & 297 & 274 & 26 & 17 & 2.8 & 0.48 \\
SD & 0.7 & 88 & 101 & 9 & 16 & 0.5 & 0.21 \\
$P$ valuet & $<.02$ & $<.02$ & $<.02$ & $<.02$ & NS & NS & $<.02$ \\
\hline
\end{tabular}

NYHA, New York Heart Association functional class; LVM, LV mass; EDV, LV volume at end-diastole; $M R F$, mitral regurgitant fraction; $\mathrm{Cl}$, cardiac index; $N S$, not statistically significant.

* Not computed because phase-contrast data was not acquired. $\dagger P$ values represent paired comparisons with baseline results.

10 studies, with 1 data set not analyzed because of the absence of baseline images. Also, data were incomplete in 1 additional post-PLV study (patient 9), in which apical tagged images were unsuitable for analysis because of electrocardiographic triggering errors (although basal and midventricular data were analyzed in this case).

\section{Function and Effects of Surgery}

LV morphologic and functional data for each patient at baseline are detailed in Table 1. The effects of PLV surgery on the 9 patients who underwent post-PLV MRI are shown in Table 2. The average decreases in LV volume at enddiastole and LV mass from baseline were $187 \pm 119 \mathrm{~mL}$ and $115 \pm 48 \mathrm{~g}$, respectively $(P<.02$ for each). No statistically significant differences existed in LV mass, LV volume at end-diastole, ejection fraction (EF), mitral regurgitant fraction, cardiac index, or $\mathrm{V}_{\mathrm{cf}}$ at baseline between those patients with post-PLV data (patients 1-9) and those patients without post-PLV data (patients 10-21; Table 1).

LV function results were mixed after PLV, with significant increases in EF and $\mathrm{V}_{\text {cf }}$ relative to baseline but no change in cardiac index (Table 2). In patients who underwent post-PLV studies, baseline EF was $16 \% \pm 10 \%$ and $\mathrm{V}_{\text {cf }}$ was $0.25 \pm 0.24 \mathrm{circ} / \mathrm{s}$. Changes in $\mathrm{V}_{\text {cf }}$ after PLV resulted from increases in both $\% \Delta \mathrm{D}(8 \% \pm 7 \%$ baseline, $14 \% \pm 6 \%$ after PLV,$P=.02)$ and RR interval (637 \pm 94 $\mathrm{ms}$ baseline, $768 \pm 84 \mathrm{~ms}$ after PLV, $P<.02$ ).

\section{Rotation}

Baseline global systolic rotation is shown in Figure 2 for each of the 21 subjects with adequate grid tagged image data. Mean baseline end-systolic rotations were $-1.9^{\circ} \pm$

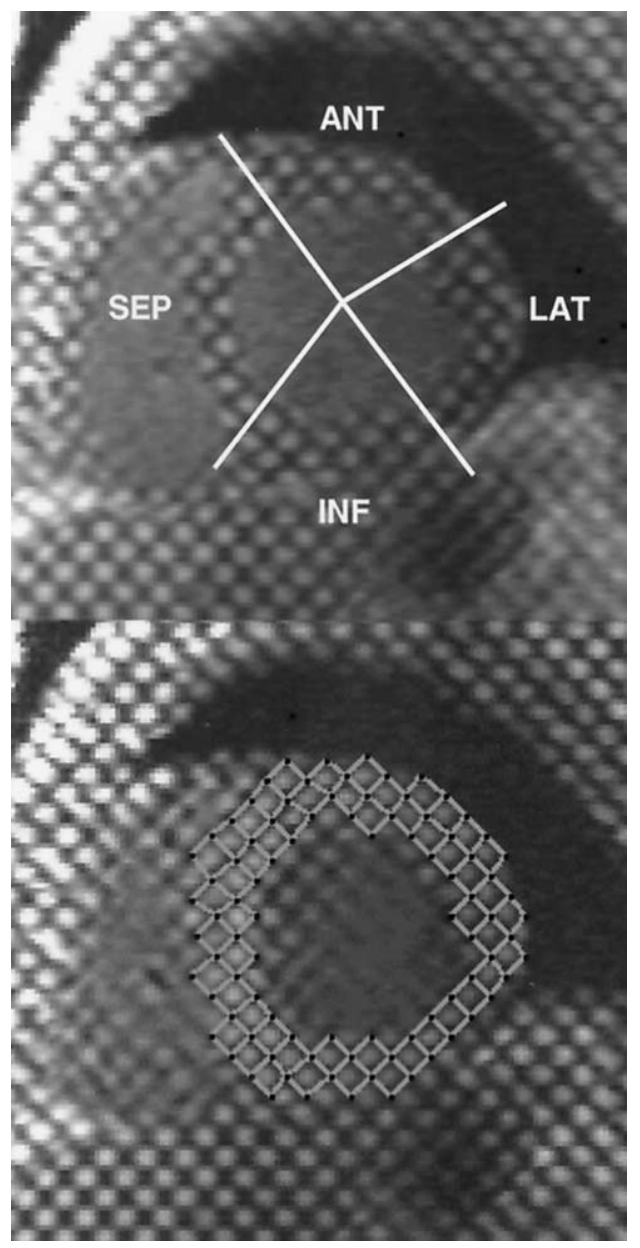

Figure 1. Midventricular tagged short-axis images from patient 1. Image at top was acquired at end-diastole and shows how basal and midventricular slices were subdivided into anterior (ANT), lateral (LAT), inferior (INF), and septal (SEP) regions. Image at bottom was acquired $\mathbf{4 5} \mathrm{ms}$ into systole and shows grid overlay used to track tag intersections during systole.

$4.3^{\circ}$ at the apex, $-2.5^{\circ} \pm 3.2^{\circ}$ at midventricle, and $-3.5^{\circ} \pm$ $2.7^{\circ}$ at the base.

Global end-systolic rotations at baseline and after PLV are displayed in Figure 3 for each of the 9 patients with data at both points, with pooled global rotation results shown in Figure 4. In these patients, mean baseline end-systolic rotations were $-3.2^{\circ} \pm 5.0^{\circ}$ at the apex, $-3.3^{\circ} \pm 3.6^{\circ}$ at midventricle, and $-3.2^{\circ} \pm 2.7^{\circ}$ at the base. Mean endsystolic rotations after PLV were $-1.4^{\circ} \pm 4.2^{\circ}$ at the apex $(P=.22),-0.4^{\circ} \pm 2.4^{\circ}$ at midventricle $(P<.02)$, and $-1.0^{\circ} \pm 2.0^{\circ}$ at the base $(P<.05)$.

Regional end-systolic rotations at baseline and after PLV are also displayed in Figure 4. End-systolic rotation was not altered significantly in the apical septal or apical free wall regions after PLV. Also, inferior wall end-systolic rotation was unchanged at the midventricular and basal levels. How- 


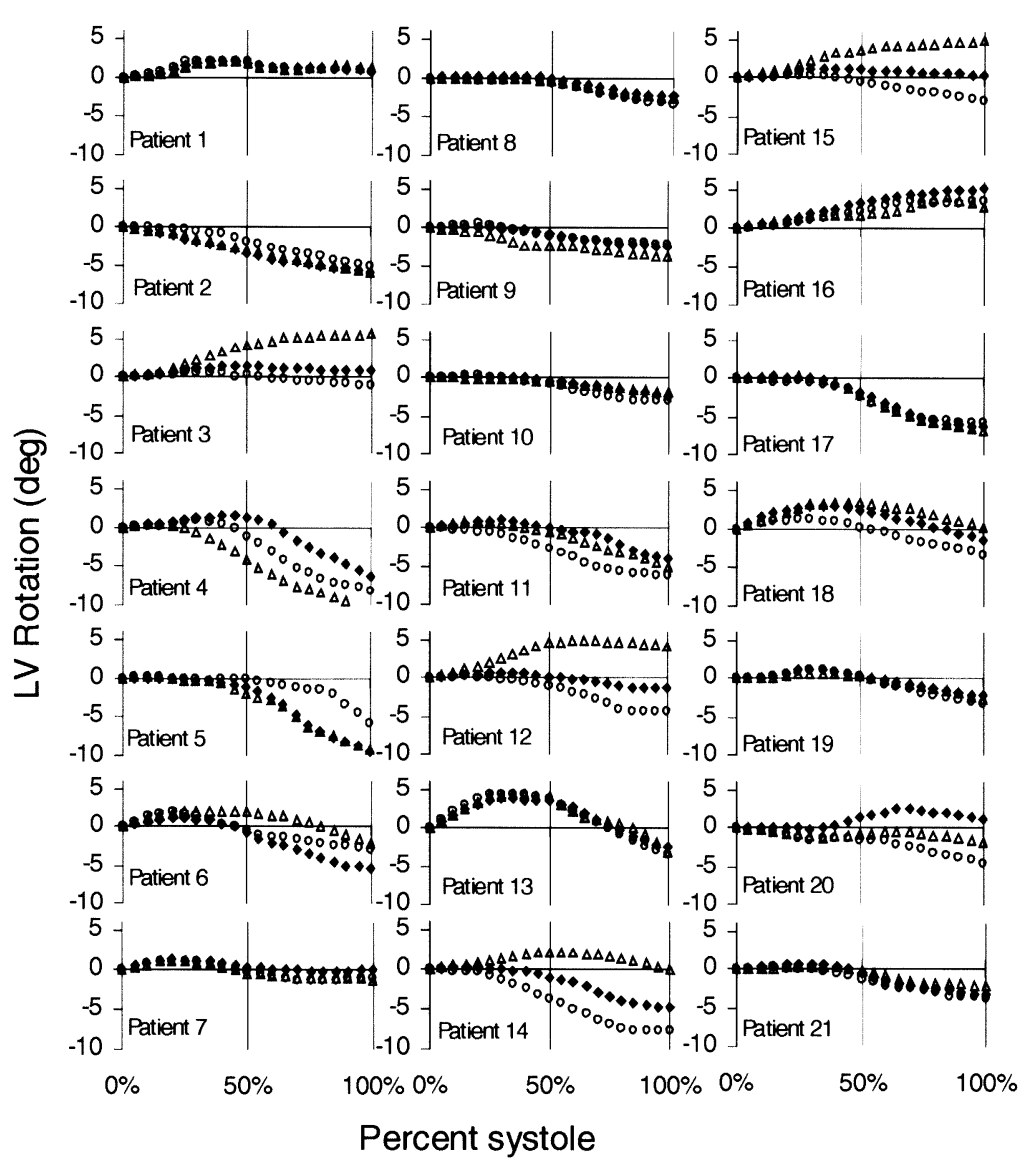

Figure 2. LV systolic rotations at apex (triangles), midventricle (diamonds), and base (circles) in all 21 patients at baseline. Patients typically exhibited identical rotation at all short-axis levels for the first approximately $20 \%$ of systole, at which point they diverged into one of two patterns: either continuation of identical rotation at all levels for the remainder of systole (eg, patient 1 ) or a divergence of rotation so the apex and base rotated in opposite directions (eg, patient 3).

ever, anterior and septal end-systolic rotations at both the midventricular and basal levels were significantly altered after PLV.

\section{Torsion}

Systolic torsion at baseline and after PLV in applicable patients is shown in Figure 5. Mean end-systolic torsion in these patients $(\mathrm{n}=8)$ was $0.0^{\circ} \pm 3.0^{\circ}$ at baseline and $-0.2^{\circ}$ $\pm 3.2^{\circ}$ after PLV $(P=.73)$. If the results from all 21 patients are considered (Table 1), the mean end-systolic torsion at baseline was $1.6^{\circ} \pm 3.5^{\circ}$.

At baseline, the relationship between end-systolic torsion and LV function was statistically significant $(P=.01$ for EF and $P<.01$ for $\mathrm{V}_{\mathrm{cf}}$ ). In patients with baseline torsion of at least $2^{\circ}$ (patients $3,12,14,15,18,20$, and 21), EF was $28 \%$ $\pm 13 \%$ and $V_{\text {cf }}$ was $1.44 \pm 0.74 \mathrm{circ} / \mathrm{s}$. In the remaining patients, however, all of whom had baseline torsion of $1^{\circ}$ or less, $\mathrm{EF}$ was $15 \% \pm 9 \%$ and $\mathrm{V}_{\mathrm{cf}}$ was $0.61 \pm 0.54 \mathrm{circ} / \mathrm{s}$.

\section{Discussion}

The counterclockwise twisting of the LV apex relative to the base (torsion) during systole is a direct result of the 3-dimensional structure of LV myocardium and is related to systolic wall thickening and the ejection of blood. ${ }^{26}$ Ventricular torsion has been studied with a variety of techniques, ranging from radiopaque markers implanted in the myocardium to cine MRI with tissue tagging. ${ }^{3-9,25-27}$ As a result, the patterns of LV rotation and torsion have been quantified in species from mouse to human being and have been shown to represent a fundamental property of mammalian hearts. ${ }^{27}$ Using mathematical models, it has been shown that torsion is necessary for uniform systolic fiber shortening across the myocardium, which may have implications for minimizing oxygen consumption., ${ }^{2,3}$

Alterations in the pattern or magnitude of systolic rotation and torsion are a sensitive indicator of myocardial disease, ${ }^{5-9}$ as recently demonstrated in a study that reported 

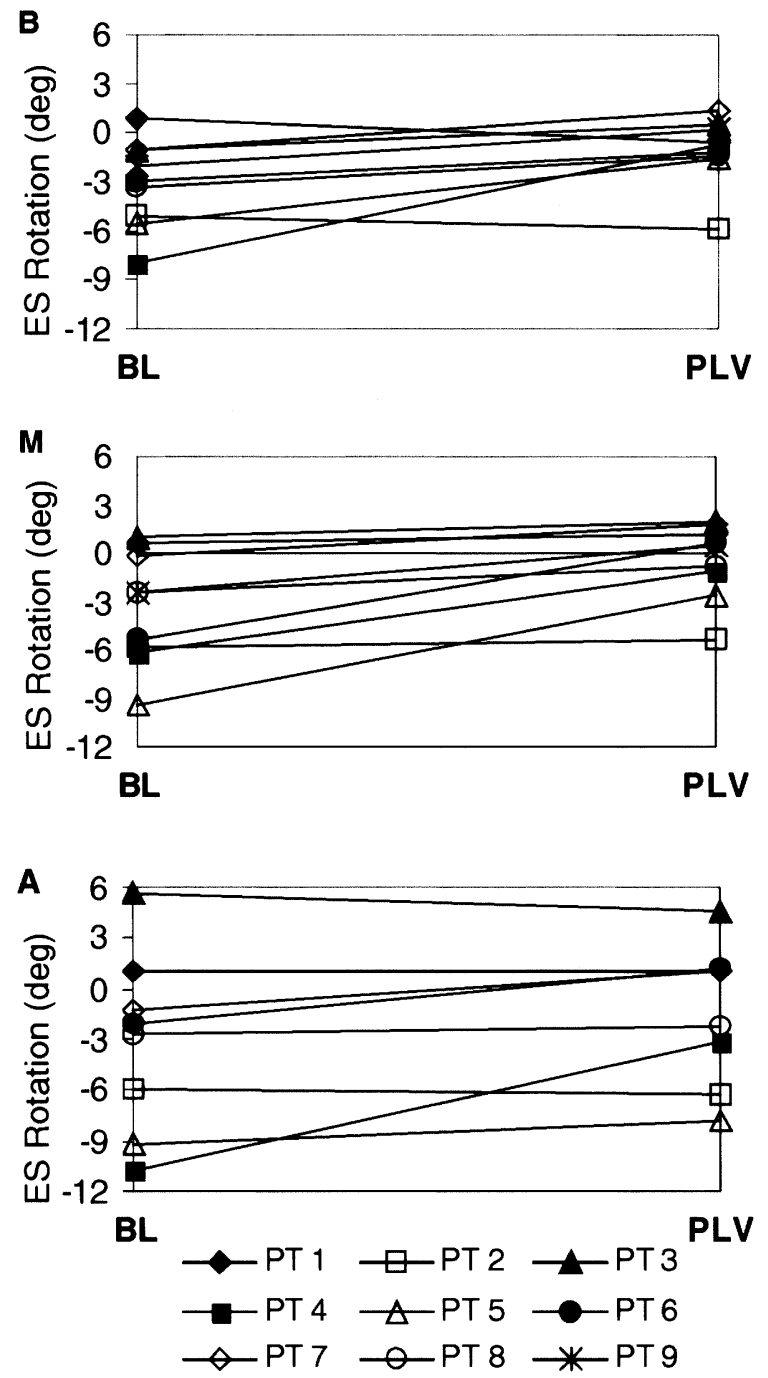

Figure 3. Changes in end-systolic (ES) rotation from baseline (BL) to after PLV (PLV) at base $(B)$, midventricle $(M)$, and apex $(A)$ for each patient (PT) imaged at both times.

diminished systolic torsion in an animal model of tachycardia-induced cardiomyopathy. ${ }^{9}$ Also, because torsion is defined as twisting of the LV apex relative to the base, changes in rotation at either short-axis level may adversely affect LV torsion. End-systolic rotation has been reported previously in healthy volunteers, with typical apical values of $9.5^{\circ}$, $6.8^{\circ}$, and $12.5^{\circ 4,5,8}$; and basal values of $-3.5^{\circ}$ and $-3.1^{\circ} .4,8$

\section{Effects of Dilated Cardiomyopathy}

In this investigation we have shown that LV torsion is severely depressed in patients with DCM relative to previously reported normal data. 3,4,6,25,27 Furthermore, we found greater end-systolic torsion values to be associated with better cardiac function in patients with DCM before PLV.

We have also shown considerable variability in LV systolic rotation among patients with DCM with otherwise
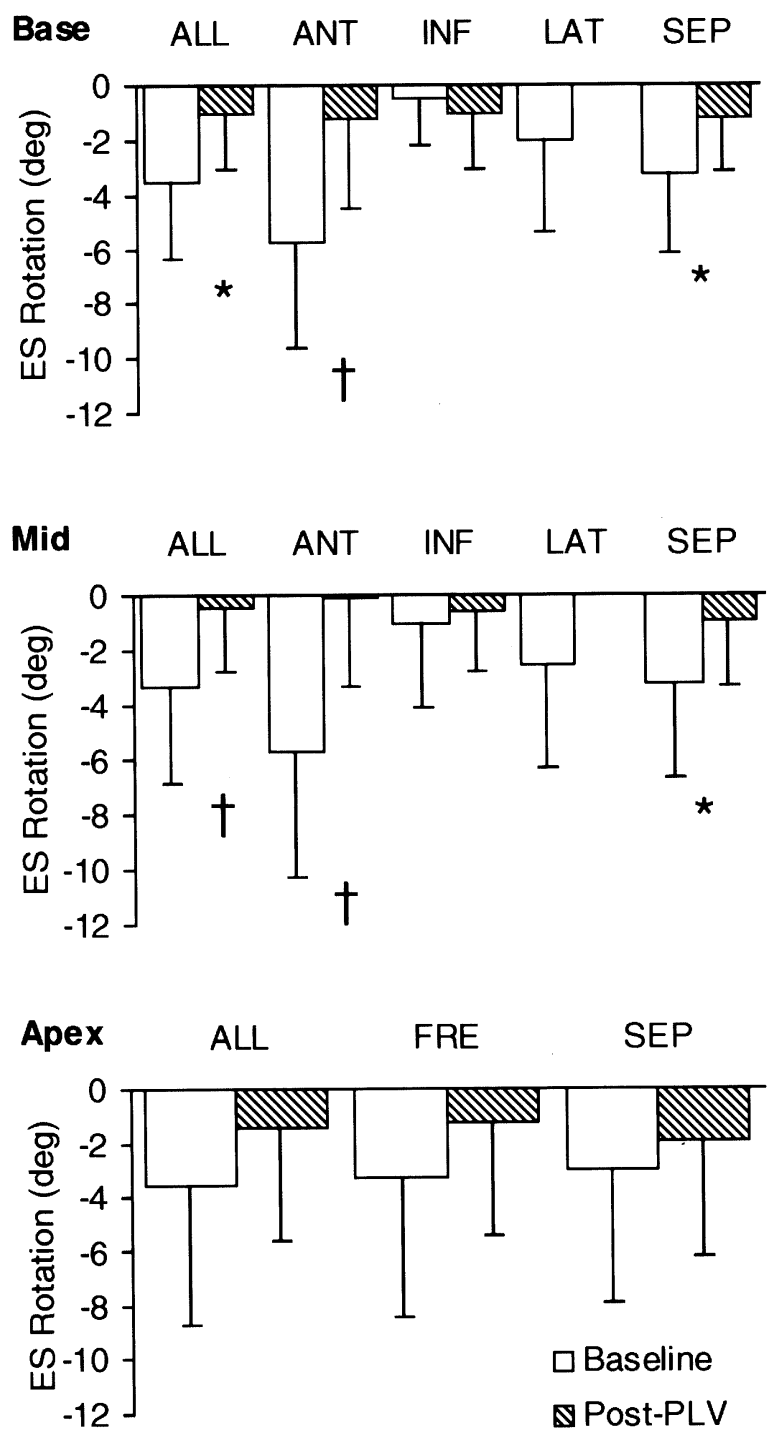

Figure 4. Pooled global and regional end-systolic (ES) rotations at base, midventricle (Mid), and apex for patients at baseline (white bars) and after PLV (striped bars). Apical results are shown for whole slice (ALL), free wall (FRE), and septum (SEP). Results for midventricle and base are shown for whole slice (ALL), anterior wall (ANT), inferior wall (INF), lateral wall (LAT), and septum (SEP). Lateral wall results are absent after PLV because this tissue was removed during surgery. Error bars represent SD. Asterisk indicates $\boldsymbol{P}<.05$ for difference in rotation at given location between baseline and post-PLV studies; dagger indicates $\boldsymbol{P}<.02$ for difference in rotation at given location between baseline and post-PLV studies.

similarly depressed cardiac function. Rotation patterns in the current study fell into 2 categories (Figure 2). In most cases the LV apex and base rotated together throughout systole, with no appreciable torsion generation. In the remaining cases the pattern of LV rotation was approximately normal at both the apex and base, ${ }^{4}$ although the magnitude of rotation was reduced at each level. 


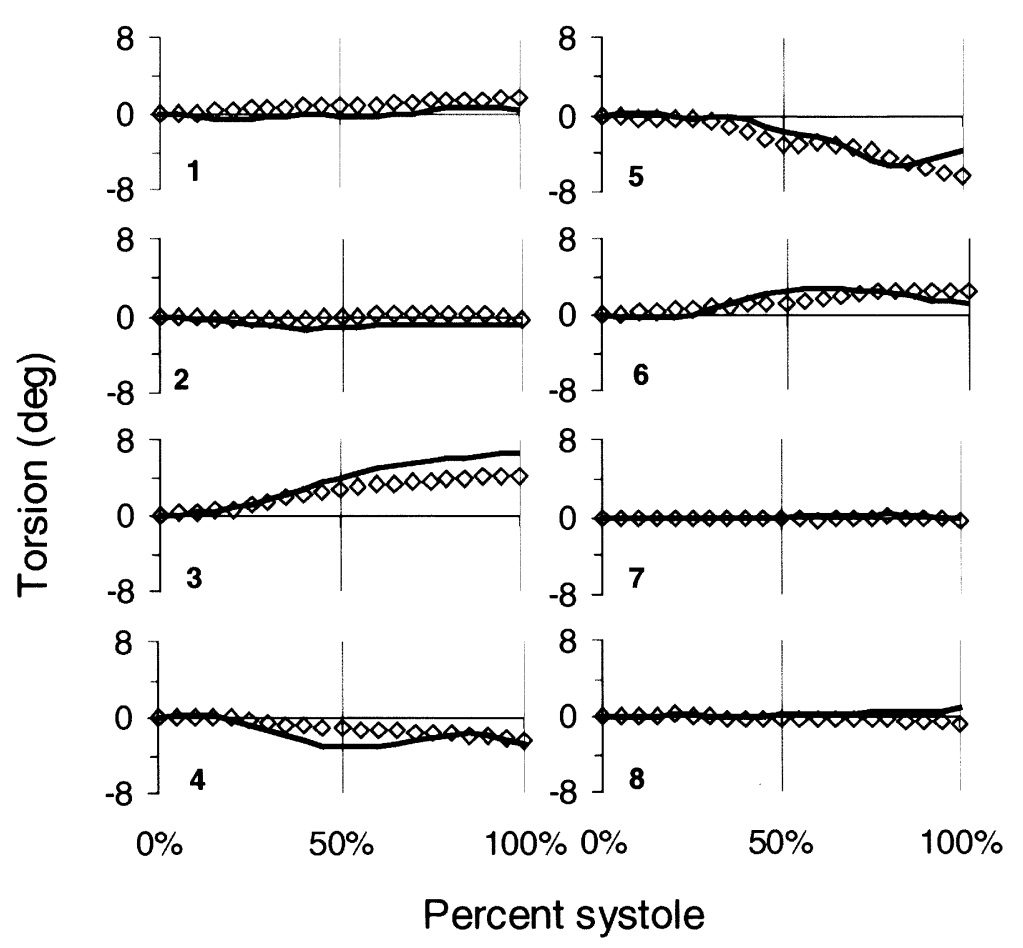

Figure 5. Systolic torsion in all patients at baseline (line) and after PLV (diamonds). Overall, torsion was unchanged after PLV, although it tended to approach zero at all systolic time points after surgery.

It is thought that LV torsion is influenced by myofiber contractility and ventricular microstructure, with the latter consisting of myofiber orientation and extracellular matrix composition and architecture. ${ }^{26}$ Myocardial fibrosis is increased in DCM, accompanied by reduced collagen tethers between myofiber bundles and a diminished collagen I/III ratio, and is associated with impaired cardiac function. ${ }^{11,28}$ It has been postulated that these factors could contribute to myofiber rearrangement and slippage ${ }^{11}$ and might influence LV torsion as well. The degree of fibrosis in DCM is variable both within and among patients, offering a possible explanation for the differences in rotation patterns observed in this study. ${ }^{28,29}$ Intrapatient fibrosis variability has also been shown to persist after PLV but is not related to patient outcome. ${ }^{14,30}$

\section{Effects of PLV}

Three months after PLV, LV torsion was unchanged from baseline (Figure 5). Furthermore, there was no significant change in end-systolic rotation at the apex, which was remote from the myectomy. However, end-systolic rotations at the base and midventricle were reduced significantly.

A trend toward zero rotation was observed in all regions and at all levels after PLV, possibly resulting from gross changes in ventricular structure caused by the surgery. This tendency was most dramatic in the anterior wall at the base and midventricle. Therefore we should not conclude that myocar- dial mechanics were unaffected by the surgery solely because torsion was unchanged. On the contrary, the observed changes in rotation indicate that myocardial mechanics were affected significantly, and only because basal and apical rotations diminished similarly was torsion unchanged after PLV.

After surgery, $\mathrm{EF}$ and $\mathrm{V}_{\mathrm{cf}}$ were significantly increased relative to baseline values, but no change in cardiac index was observed. Thus, despite the lack of improvement in myocardial mechanics, some ejection phase indices of LV function did improve. However, this could simply reflect changes in LV geometry caused by surgery, with no actual improvement in systolic function.

\section{Limitations}

Limitations of this study include the small number of patients studied after PLV. Many patients were unavailable for study by MRI after surgery. In addition, our post-PLV examinations were biased toward patients in relatively better health and with more favorable outcomes than the PLV population in general. Furthermore, we only used loaddependent indices to assess LV function.

In some cases portions of the LV wall were only a single tag width thick, which added uncertainty to the rotation measurements. Through-plane motion of short-axis slices during systole has been shown to be significant in healthy patients and was not accounted for in this study.$^{31}$ However, mitral valve plane motion was minimal in these patients (the 
average long-axis length change during systole was less than a single slice thickness), and we therefore do not consider this factor to have significantly affected our results. Finally, in some cases we had difficulty defining the anterior-inferior wall junction after PLV, which along with the inclusion of scar tissue from this region in our analysis may have negatively affected our results.

\section{Conclusions}

$\mathrm{LV}$ rotation and torsion are impaired in DCM, possibly as a result of diminished myocardial contractility and increased fibrosis. However, considerable heterogeneity in rotation patterns exists among patients with DCM. PLV caused significant regional changes in end-systolic rotation at basal and midventricular levels, coinciding with the myocardial excision, but apical values were unchanged. We believe that cardiac MRI with dynamic tissue tagging provides valuable insight into the function of the LV in patients with DCM and can be applied to planning and monitoring their responses to both medical and surgical therapies. In addition, it can be used for noninvasive assessment of the efficacy of new therapeutic procedures under consideration for the treatment of congestive heart failure.

We thank Bernhard Sturm, PhD, Tiffany Buda, RN, and Christine Lorenz, $\mathrm{PhD}$, for their contributions to this article.

\section{References}

1. Streeter DD. Fiber orientation in the canine left ventricle during systole and diastole. Circ Res. 1969;24:339-47.

2. Arts T, Reneman RS, Veenstra VC. A model of the mechanics of the left ventricle. Ann Biomed Eng. 1979;7:299-318.

3. Buchalter MB, Weiss JL, Rogers WJ, Zerhouni EA, Weisfeldt ML, Beyar R, et al. Noninvasive quantification of left ventricular rotational deformation in normal humans using magnetic resonance imaging myocardial tagging. Circulation. 1990;81:1236-44.

4. Lorenz CH, Pastorek JS, Bundy JM. Delineation of normal human left ventricular twist throughout systole by tagged cine magnetic resonance imaging. J Cardiovasc Magn Reson. 2000;2:97-108.

5. Stuber M, Scheidegger MB, Fischer SE, Nagel E, Steinnermann F, Hess OM, et al. Alterations in the local myocardial motion pattern in patients suffering from pressure overload due to aortic stenosis. Circulation. 1999;100:361-8.

6. Hansen DE, Daughters GT, Alderman EL, Stinson EB, Baldwin JC, Miller DC. Effect of acute cardiac allograft rejection on left ventricular systolic torsion and diastolic recoil measured by intramyocardial markers. Circulation. 1987;76:988-1008.

7. Young AA, Dokos S, Powell KA, Sturm B, McCulloch AD, Starling $\mathrm{RC}$, et al. Regional heterogeneity of function in nonischemic dilated cardiomyopathy. Cardiovasc Res. 2001;49:308-18.

8. Nagel E, Stuber M, Lakatos M, Scheidegger MB, Boesiger P, Hess OM. Cardiac rotation and relaxation after anterolateral myocardial infarction. Coron Artery Dis. 2000;11:261-7.

9. Tibayan FA, Lai DT, Timek TA, Dagum P, Liang D, Daughters GT, et al. Alterations in left ventricular torsion in tachycardia-induced dilated cardiomyopathy. $J$ Thorac Cardiovasc Surg. 2002;124:43-9.

10. MacGowan GA, Shapiro EP, Azhari H, Siu CO, Hees PS, Hutchins $\mathrm{GM}$, et al. Noninvasive measurement of shortening in the fiber and cross-fiber directions in the normal human left ventricle and in idiopathic dilated cardiomyopathy. Circulation. 1997;96:535-41.
11. Weber KT. Cardiac interstitium in health and disease: the fibrillar collagen network. J Am Coll Cardiol. 1989;13:1637-52.

12. Batista RJ, Santos JL, Takeshita N, Bocchino L, Lima PN, Cunha MA. Partial left ventriculectomy to improve left ventricular function in end-stage heart disease. J Card Surg. 1996;11:96-7.

13. Franco-Cereceda A, McCarthy PM, Blackstone EH, Hoercher KJ, White JA, Young JB, et al. Partial left ventriculectomy for dilated cardiomyopathy: is this an alternative to transplantation? J Thorac Cardiovasc Surg. 2001;121:879-93.

14. Higuchi ML, Moreira LF, Silvestre JM, Gutierrez PS, Savalli C, Stolf $\mathrm{N}$, et al. Myocardial fiber diameter as a good indicator of outcome in Batista's operation. J Card Surg. 1999;14:401-7.

15. Starling RC, McCarthy PM, Buda T, Wong J, Goormastic M, Smedira NG, et al. Results of partial left ventriculectomy for dilated cardiomyopathy: hemodynamic, clinical and echocardiographic observations. J Am Coll Cardiol. 2000;36:2098-103.

16. Dickstein ML, Spotnitz HM, Rose EA, Burkhoff D. Heart reduction surgery: an analysis of the impact on cardiac function. $J$ Thorac Cardiovasc Surg. 1997;113:1032-40.

17. Ratcliffe MB, Hong J, Salahieh A, Ruch S, Wallace AW. The effect of ventricular volume reduction surgery in the dilated, poorly contractile left ventricle: a simple finite element analysis. $J$ Thorac Cardiovasc Surg. 1998;116:566-77.

18. McCarthy PM, Starling RC, Wong J, Scalia GM, Buda T, Vargo RL, et al. Early results with partial left ventriculectomy. J Thorac Cardiovasc Surg. 1997;114:755-65.

19. DeVega NG. La annuloplastia selectiva regulable y permanete: una tecnica original para el tratamiento de la insufficiencia tricuspide. Rev Esp Cardiol. 1972;25:555-6.

20. Foo TK, Bernstein MA, Aisen AM, Hernandez RJ, Collick BD, Bernstein T. Improved ejection fraction and flow velocity estimates with use of view sharing and uniform repetition time excitation with fast cardiac techniques. Radiology. 1995;195:471-8.

21. Axel L, Dougherty L. Heart wall motion: improved method of spatial modulation of magnetization for MR imaging. Radiology. 1989;172: 349-50.

22. Firmin DN, Nayler GL, Klipstein RH, Underwood SR, Rees RS, et al. In vivo validation of MR velocity imaging. J Comput Assist Tomogr. 1987;11:751-6.

23. Quinones MA, Gaasch WH, Alexander JK. Influence of acute changes in preload, afterload, contractile state and heart rate on ejection and isovolumic indices of myocardial contractility in man. Circulation. 1976;53:293-302.

24. Glantz SA. Primer of biostatistics. 5th ed. New York: McGraw-Hill, 2002.

25. Young AA, Kramer CM, Ferrari VA, Axel L, Reichek N. Threedimensional left ventricular deformation in hypertrophic cardiomyopathy. Circulation. 1994;90:854-67.

26. Ingels NB, Hansen DE, Daughters GT, Stinson EB, Alderman EL, Miller DC. Relation between longitudinal, circumferential, and oblique shortening and torsional deformation in the left ventricle of the transplanted human heart. Circ Res. 1989;64:915-27.

27. Henson RE, Song SK, Pastorek JS, Ackerman JJ, Lorenz CH. Left ventricular torsion is equal in mice and humans. Am J Physiol Heart Circ Physiol. 2000;278:H1117-23.

28. Schwarz F, Mall G, Zebe H, Blickle J, Derks H, Manthey J, et al. Quantitative morphologic findings of the myocardium in idiopathic dilated cardiomyopathy. Am J Cardiol. 1983;51:501-6.

29. Frazier OH, Gradinac S, Segura AM, Przybylowski P, Popovic Z, Vasilijevic J, et al. Partial left ventriculectomy: Which patients can be expected to benefit? Ann Thorac Surg. 2000;69:1836-41.

30. Cury PM, Higuchi ML, Gutierrez PS, Moreira LF, Bocchi EA, Stolf NA, Jatene AD. Autopsy findings in early and late postoperative death after partial left ventriculectomy. Ann Thorac Surg. 2000;69:769-73.

31. Pattynama PM, Doornbos J, Hermans J, van der Wall EE, de Roos A. Magnetic resonance evaluation of regional left ventricular function: effect of through-plane motion. Invest Radiol. 1992;27:681-5. 